\title{
Megakaryocyte biology and related disorders
}

\author{
Liyan Pang, Mitchell J. Weiss, and Mortimer Poncz
}

\author{
Division of Hematology, Children's Hospital of Philadelphia, and Department of Pediatrics, University of Pennsylvania School of Medicine, \\ Philadelphia, Pennsylvania, USA.
}

\begin{abstract}
Platelets, derived from megakaryocytes, have an essential role in thrombosis and hemostasis. Over the past 10 years, a great deal of new information has been obtained concerning the various aspects of hematopoiesis necessary to maintain a steady-state platelet level to support physiologic hemostasis. Here we discuss the differentiation of HSCs into megakaryocytes, with emphasis on the key cytokine signaling pathways and hematopoietic transcription factors. Recent insight into these processes elucidates the molecular bases of numerous acquired and inherited hematologic disorders. It is anticipated that the growing knowledge in these areas may be exploited for new therapeutic strategies to modulate both platelet numbers and their thrombogenicity.
\end{abstract}

\section{Development of the megakaryocyte lineage from HSCs}

Like all terminally differentiated hematopoietic cells, megakaryocytes arise from common HSCs, which are responsible for lifelong production of all circulating blood cells (1). Hematopoietic cells are classified by 3 means: (a) by surface markers that are mainly detected by flow cytometry, (b) by their developmental potential assessed ex vivo in colony assays, and (c) by their ability to reconstitute host animals in vivo. Individual cells that reconstitute multilineage hematopoiesis for at least 6 months, termed long-term repopulating HSCs, are rare, constituting less than $0.1 \%$ of total nucleated marrow cells. In mice, these are highly enriched within a population of cells with surface markers $\mathrm{Lin}^{-} \mathrm{Sca}-1^{+} \mathrm{c}-\mathrm{kit} \mathrm{t}^{\text {high }}(2-5)$ (Figure 1). This population has been referred to as the LSK longterm HSC population, but for ease of reading we will refer to it below as the "HSC" population. The production of mature blood cells from HSCs involves a series of successive differentiation steps in which the developmental and proliferative capacities of progenitors become increasingly restricted.

The differentiation of HSCs has been tracked by the expression of cell surface markers including the tyrosine kinase cytokine receptor Flt3 (6), which is absent on the HSC (7). The classical model for hematopoiesis is that committed HSCs give rise to 2 lineages, a common lymphoid progenitor capable of producing lymphocytes, and a common myeloid progenitor with developmental potential restricted to myeloid, macrophage, eosinophil, erythroid, and megakaryocyte lineages (Figure 1) (8-10). Erythroid and megakaryocyte lineages arise from a common megakaryocyte-erythroid progenitor (MEP) derived from the common myeloid progenitor (11). However, recent studies reveal that progenitors that have surface markers similar to those of HSCs, but have become Flt3-positive, upon further differentiation into lymphoid and myeloid lineages do not produce megakaryocytes or erythrocytes in vitro or in vivo (Figure 1) (12). Thus FLT3-negative HSCs express markers of committed megakaryocytes and erythroid precursors and may directly give rise to MEPs (see below and Figure 1). When early stem cells become Flt $3^{+}$, erythromegakaryocytic marker expression is lost, while lymphoid and myeloid

Nonstandard abbreviations used: CBFA2, core-binding factor $\alpha-2$; EPO, erythropoietin; ET, essential thrombocythemia; MEP, megakaryocyte-erythroid progenitor; PF4, platelet factor 4; SDF-1, stromal cell-derived factor-1; TPO, thrombopoietin. Conflict of interest: The authors have declared that no conflict of interest exists.

Citation for this article: J. Clin. Invest. 115:3332-3338 (2005). doi:10.1172/JCI26720. marker expression and developmental potential are retained. These findings deviate from the classical model for hematopoiesis and indicate that loss of erythromegakaryocytic potential may represent a relatively early event in HSC differentiation in certain study models. This surprising finding highlights our incomplete understanding of hematopoiesis and the plasticity of the process. The model presented in Figure 1 takes into account both the classical pathway, which predicts that HSCs split into common myeloid and lymphoid progenitors (8-10), and the newer findings suggesting a direct pathway from the HSC to the MEP (12). Improvement in fine mapping of cell lineages by flow cytometry analysis and the development of new approaches for studies of the earliest stages of hematopoiesis may further delineate the steps involved in lineage commitment under varied circumstances.

While erythroid and megakaryocyte lineages are believed to share a common MEP (8-10) (Figure 1), the signals that regulate the final separation of these lineages are not well understood. Erythroid and megakaryocytic precursors express both common and unique hematopoietic transcription factors. Among the latter, no single unique factors have been identified to determine lineage choice of the MEP. It is also possible that the final lineage of the MEP is determined by the combinatorial action of multiple nuclear proteins.

The first cells fully committed to the megakaryocyte lineage, termed CFU-Meg, are characterized by a unique cell surface phenotype (13) and form a small cluster of pure megakaryocytes in culture. CFU-Meg cells give rise to $2 \mathrm{~N}$ megakaryocytes, which, in turn, undergo endomitosis and cytoplasmic differentiation, resulting in a pool of mature megakaryocytes recognized by their large size and characteristic morphology. In the normal human marrow, approximately 1 in 10,000 nucleated cells is a megakaryocyte, while in disorders associated with increased peripheral platelet destruction, such as immune thrombocytopenia purpura, the number increases about 10 -fold (14).

Megakaryopoiesis is first noted in the embryonic yolk sac, although studies of animals with severe quantitative and qualitative platelet deficiencies, such as NF-E2-/- mice, showed that platelets are not critical for prenatal survival (15). In mice, fetal megakaryocytes appear to represent a distinct lineage with biologic features different from those of their adult counterparts (16). Several human disorders also support this point. One example is the thrombocytopenia and absent radius (TAR) syndrome, a disorder of unknown etiology characterized by moderate to severe thrombocytopenia in infancy that is typically outgrown in early 


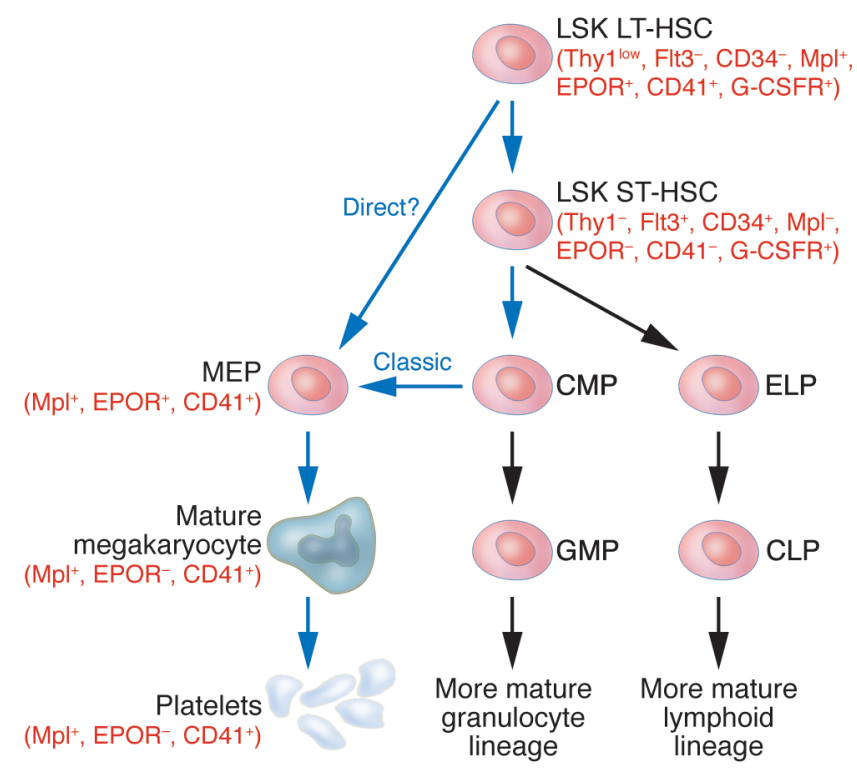

Figure 1

Megakaryopoiesis pathways. The figure extends from the HSC to platelets and offers a combination of the more "classical" pathway, leading to the common megakaryocyte-erythroid progenitor (MEP), and a proposed "direct" route from the HSC. Pathways leading to platelet production are indicated by blue arrows and other pathways by gray arrows. Surface markers of importance are noted in parentheses in red. LT-HSC, long-term HSC; ST-HSC, short-term HSC; Thy1, thymus 1 ("low" indicates low surface antigen and "-" indicates none detectable); FIt3, FMS-like tyrosine kinase 3; EPOR, erythropoietin receptor; CD41, glycoprotein IIb/IIla or $\alpha_{\| \mathrm{lb}} \beta_{3}$ integrin receptor; G-CSFR, G-CSF receptor; CMP, common myeloid progenitor; ELP, early lymphoid progenitor; GMP, granulocyte/monocyte progenitor; CLP, common lymphoid progenitor.

childhood (17). Another example is transient myeloproliferative disorder and acute megakaryoblastic leukemia in Down syndrome, which develop nearly exclusively in the neonatal period and the first years of life, respectively (18). Interestingly, recent studies demonstrate that fetal megakaryocyte progenitors are uniquely sensitive to mutations in the transcription factor GATA-1, which accompany these disorders (19). Perhaps another example of the distinct nature of fetal/infant megakaryopoiesis is the well-known propensity of severely ill neonates to develop prolonged thrombocytopenia with slow marrow recovery of platelet production (20).

\section{Unique aspects of megakaryocyte maturation}

The hallmark of megakaryocyte development is the formation of a large cell $(\sim 50-100 \mu \mathrm{m}$ diameter) containing a single, large, multilobulated, polyploid nucleus (21). Eventually, each megakaryocyte releases approximately $10^{4}$ platelets (22). Unlike other cells, megakaryocytes undergo an endomitotic cell cycle during which they replicate DNA but do not undergo anaphase or cytokinesis; as a result, they acquire a DNA content of up to $256 \mathrm{~N}$ per cell (23). The mechanisms regulating endomitosis are not fully understood. Clearly cyclins are involved, though a combined knockout of cyclins D1, D2, and D3, while specifically affecting hematopoiesis and causing late midgestation fetal loss due in part to anemia, was not noted to affect megakaryopoiesis (24). On the other hand, the cyclin E-null mouse clearly had a defect in megakaryopoiesis and in development of trophoblasts, another cell line dependent on endomitosis (25). Other studies on chromosomal passenger proteins Aurora-B, survivin, and inner centromere protein showed normal levels overall in megakaryocytes (26), although one report suggests that survivin and Aurora-B may be mislocalized or absent during an important phase of endomitosis (27). The biologic importance of endoreduplication is unclear in terms of its necessity for cell size and for platelet release.

Cellular maturation of megakaryocytes is distinguished by accumulation of characteristic surface markers including $\mathrm{GP}_{\mathrm{Ib}} \alpha, \beta / \mathrm{GPIX} / \mathrm{GPV}$ receptors, a cytoplasmic demarcation system believed to participate in platelet formation, distinctive platelet organelles such as the $\alpha$ - and dense granules, and organelle granular proteins that participate in platelet function, such as platelet factor 4 (PF4) and vWF (28). Of note, the extent of polyploidization is not closely synchronized with cellular maturation, so that different degrees of ploidy are present at each stage. For this reason it has been hard to distinguish "early-onset" megakaryocyte-specific genes from "late-onset" ones (29).

Despite their close relationship in hematopoietic phylogeny and numerous common hematopoietic transcription factors, erythroid and megakaryocyte lineages do not share many specific proteins or organelles. However, it is interesting to note that both lineages circulate in anucleate forms. The platelet equivalent in fish is called a thrombocyte and is a circulating nucleated diploid cell $(30,31)$. The purpose of platelet and erythrocyte enucleation in mammals is unclear. One possibility is that loss of nuclei increases flexibility and distensibility of circulating cells, optimizing delivery of specialized functions within small-caliber capillary beds.

\section{Cytokines involved in megakaryopoiesis}

In humans, homeostatic mechanisms regulate the normal platelet count within an approximately 3 -fold range $\left(150 \times 10^{3}\right.$ to $450 \times 10^{3}$ per cubic micrometer). Disorders that consume platelets increase their production. Numerous hematopoietic growth factors regulate different aspects of megakaryocyte biology (Figure 2). Certain cytokines, including GM-CSF, IL-3, IL-6, IL-11, IL-12, and erythropoietin (EPO), stimulate proliferation of megakaryocytic progenitors (32). Other cytokines, including IL-1 $\alpha$ and leukemia inhibitory factor (LIF), modulate megakaryocyte maturation and platelet release $(32,33)$. Many of these cytokines have broad effects on all hematopoietic lineages. Presently, the multilineage cytokine IL-11 (Neumega) is the only clinically approved drug for treating thrombocytopenia (34).

More than 10 years ago, a more potent and relatively specific megakaryocyte/platelet cytokine, termed $\mathrm{Mpl}$ ligand or thrombopoietin (TPO), was identified. This cytokine is discussed below, along with 2 chemokines, stromal cell-derived factor-1 (SDF-1; CXCL12) and PF4 (CXCL4), that have important effects on megakaryopoiesis and platelet production. A detailed Review by Kenneth Kaushansky that focuses on the role of TPO and its receptor in thrombopoiesis is part of this series on the biology of megakaryocytes and platelets (35).

TPO. $\mathrm{Mpl}$ is a GP130 family member previously identified as important for megakaryocyte formation in vitro (36). This pivotal observation led to identification of the Mpl ligand, termed TPO, which was determined to markedly stimulate megakaryocyte production (37-40). TPO is highly homologous to EPO in its N-terminal half, reflecting a close evolutionary relationship between their respective receptor signaling pathways. Abrogation of either $\mathrm{Mpl}$ 
Transcription factors

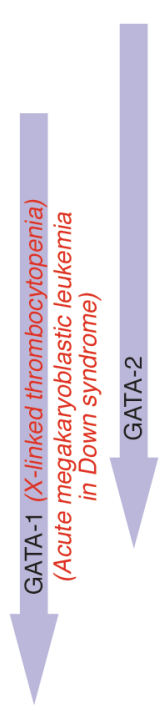

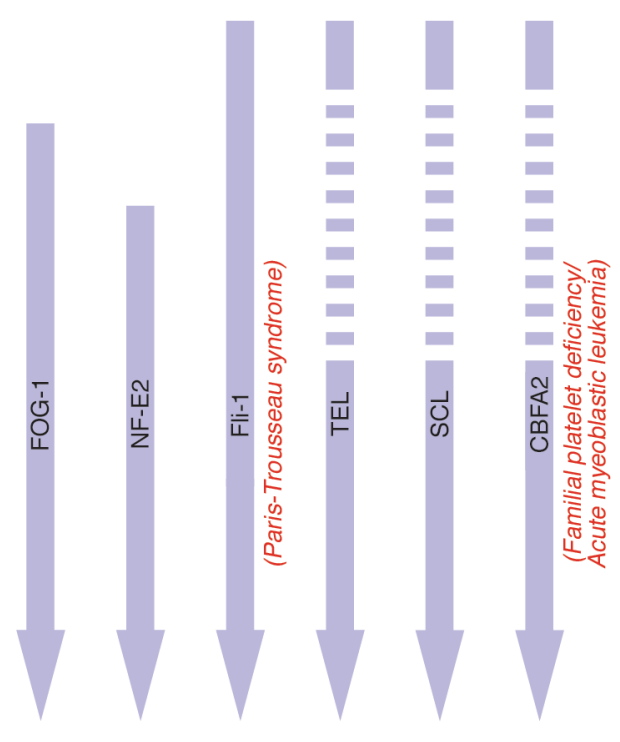

Megakaryopoiesis
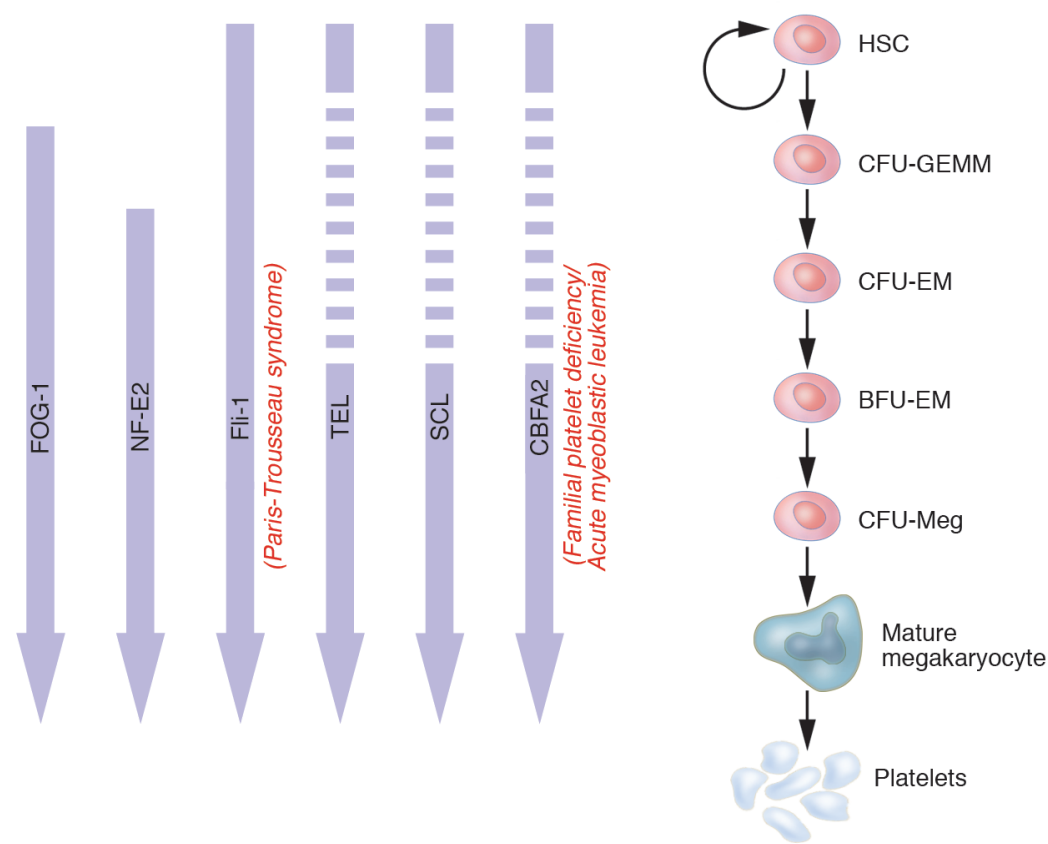

Cytokines and chemokines
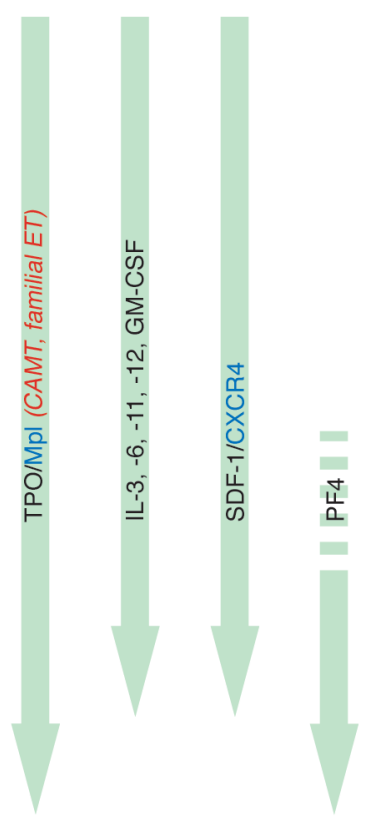

Figure 2

Regulation of megakaryopoiesis by cytokines, chemokines, and transcription factors. In the middle panel, a scheme based on the classical pathway of megakaryopoiesis is shown. Cytokines and chemokines that influence that process are shown on the right side as green arrows to indicate the approximate level of development at which they have their influence. Open white areas in arrows indicate levels at which the cytokine is not known to act. Blue text refers to cytokine receptors of significance in megakaryopoiesis. Transcription factors that affect megakaryopoiesis are shown on the left side, and the lilac-colored arrows indicate the approximate point of their influence. Open white areas in arrows indicate levels at which the transcription factor is not known to act. Clinically relevant diseases linked to defects of these regulators are noted in red, italicized text. CFU-GEMM, CFU, granulocyte, erythrocyte, macrophage, megakaryocyte; CFU-EM, CFU, erythrocyte, megakaryocyte; BFU-EM, burst-forming unit, erythrocyte, megakaryocyte; CFU-Meg, CFU, megakaryocyte.

or TPO in mice decreases megakaryocyte numbers in the marrow and circulating platelets by approximately $85 \%$ (41-43). Clearly the TPO:Mpl axis is important but not essential for megakaryopoiesis. Furthermore, studies of $\mathrm{Mpl}$ knockout animals showed that the TPO:Mpl axis functions in early hematopoietic progenitors, including HSCs $(44,45)$ (Figure 1). Thus, the TPO:Mpl axis appears to be important for hematopoiesis in general and megakaryopoiesis specifically. The discovery of TPO has contributed greatly to platelet biology, because it permits relatively large and pure cultures of megakaryocytes to be generated in vitro. TPO remains under development as a potential clinical thrombopoietic and/or hematopoietic agent and as a drug to stimulate ex vivo expansion of HSCs (46).

In adults, humoral regulation of thrombopoiesis differs from that of erythropoiesis, where the kidneys produce EPO in response to tissue hypoxia. In contrast, TPO is produced constitutively, and its circulating levels are regulated by its end product, platelets. Circulating TPO is believed to control endogenous megakaryocyte numbers and platelet count. In the steady state, TPO is synthesized predominantly and constitutively in the liver (47). $\mathrm{Mpl}$ receptors on circulating platelets absorb TPO to negatively regulate its availability for stimulating hematopoietic progenitor cells in the marrow (48). TPO is also produced by bone marrow stromal cells (49). The relative impact of circulating versus paracrine TPO production on platelet numbers is unclear. It is possible that these different modes of production satisfy distinct hematopoietic pools in different niches.
Defects in TPO:Mpl signaling occur in several human disorders. For example, Mpl mutations, mostly causing frameshifts and early termination, occur in congenital amegakaryocytic thrombocytopenia, a rare disorder of life-threatening thrombocytopenia and megakaryocyte deficiency in infancy (50-52) (Figure 2). Given the role of $\mathrm{Mpl}$ in HSC development, it is also possible that congenital amegakaryocytic thrombocytopenia patients are at risk for developing more diffuse hematopoietic defects, including aplastic anemia (53). Activating mutations in the TPO gene promoter (54) and the Mpl protein (55) occur in a subset of patients with familial essential thrombocythemia (ET) (Figure 2), a disorder characterized by increased numbers of hyperaggregable platelets. In contrast, the majority of patients with the more common acquired adult, myeloproliferative form of ET harbor somatic activating mutations in the JAK2 gene (56). These mechanistic differences could explain why familial ET carries an excellent long-term prognosis that differs from the high incidence of leukemic transformation in acquired ET (57).

SDF-1. SDF-1 enhances both megakaryopoiesis and homing of HSCs to the bone marrow during fetal development (58). SDF-1 stimulates megakaryopoiesis via TPO-independent CXCR4 receptor pathways by enhancing the chemotactic activity of their progenitors $(59,60)$. This activity of SDF-1 may be important for the movement of megakaryocyte progenitors from the proliferative "osteoblastic niche" to the "vascular niche" for platelet formation (61). Indeed, both in $\mathrm{TPO}^{-/-}$and in $\mathrm{Mpl}^{l_{-}-}$mice, infusions of SDF-1 can rescue platelet production (61). There may be clinical utility for 


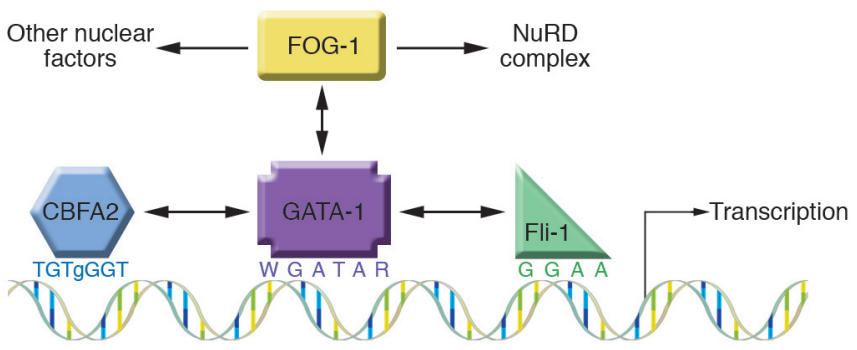

Figure 3

Assembly of transcription factor complex at a megakaryocyte-specific gene. A schematic representation of the proximal promoter region of a hypothetical megakaryocyte-specific gene modeled after the Itga2 $b$ gene. GATA- 1 is shown in the middle binding to its known consensus sequence (69) and interacting with FOG-1, Fli-1, and CBFA2 of the RUNX complex (each interaction is indicated by a 2-headed arrow). Fli-1 and CBFA2 bind to their adjacent cognate on the DNA $(89,122)$, while FOG-1 is recruited through its interactions with the $\mathrm{N}$-terminal zinc finger of GATA-1. In turn, FOG-1 recruits the NuRD complex and other nuclear factors.

SDF-1 infusions to improve platelet production. For example, HIV may cause thrombocytopenia by infecting megakaryocyte precursors through interactions with their CXCR4 receptors (62). Thus, SDF-1 mimetic drugs may improve HIV-related thrombocytopenia by competing with the virus for its megakaryocyte receptor (63).

PF4 and other chemokines. PF4 is an $\alpha$-granule protein that inhibits megakaryocyte development and maturation in vitro (64), as other CXC and CC subfamily chemokines have subsequently been shown to do (65). The in vitro findings for PF4 are corroborated by altered platelet counts in $\mathrm{PF}^{-/-}$mice and transgenic PF4-overexpressing mice (66). Platelet $\alpha$-granules contain large stores not only of the platelet-specific chemokines PF4 and the closely related protein platelet basic protein (PBP; CXCL7), but also of other chemokines, especially RANTES (CCL5) and ENA-78 (CXCL5) $(67,68)$. They negatively regulate megakaryopoiesis and are mild platelet agonists through cognate receptors on developing megakaryocytes. These are weak agonists of platelet activation and also may be important in linking thrombosis and inflammation. Release of $\alpha$-granular contents in the marrow could affect platelet numbers in pathologic states. For example, the discharge of chemokines during chemotherapy or radiation therapy may contribute to thrombocytopenia that occurs during these treatment modalities. In this case, strategies to inhibit this process could be used preemptively to prevent thrombocytopenia.

\section{Transcription factors involved in megakaryopoiesis}

Megakaryopoiesis is regulated by multiple cytokines influencing the survival and proliferation of increasingly committed progenitors as they transition from one hematopoietic niche to another in an organized fashion (61). During this process, a series of transcription factors coordinately regulate the chromatin organization of megakaryocyte-specific genes and prime them for expression en route to platelet formation. Some of these events are beginning to be understood, especially the hematopoietic-specific transcription factor complexes involved in terminal differentiation. Numerous nuclear proteins with important roles in megakaryocyte formation, growth regulation, and platelet release have been identified, mainly through loss-of-function studies in mice and analysis of human diseases. Important examples are discussed below.
GATA-1/FOG-1 complex. GATA-1 was first isolated as an essential 2-zinc finger, erythroid transcription factor that binds the DNA sequence WGATAR (69) (Figure 3). GATA-1 is also expressed and of functional consequence in megakaryocytes, mast cells, and eosinophils $(70,71)$. Transient expression reporter gene studies of megakaryocyte-specific proximal promoters defined several functionally important GATA-binding sites (72-74). A point mutation in a GATA-binding site of the GP1bb proximal promoter region causes a form of Bernard-Soulier syndrome (74).

While targeted disruption of the GATA-1 gene in mice causes embryonic lethality due to anemia (75), a megakaryocyte-specific knock down of GATA-1 expression results in significant thrombocytopenia and increased numbers of immature and dysmorphic megakaryocytes (76). GATA-2 is a closely related transcription factor and is also hematopoietic-specific, but it is expressed earlier and participates in maintenance of HSCs and multipotential progenitors (77). GATA-1 and GATA-2 are believed to have both overlapping and unique functions $(69,78)$. Continued GATA-2 expression during early megakaryopoiesis may explain the partial ability for platelet formation in the GATA-1 knockdown mouse (Figure 2).

FOG-1 (Friend of GATA-1, or Zfpm1) is a 9-zinc finger, hematopoietic-specific transcription factor isolated as a GATA-1 binding partner (79) (Figure 3). Targeted disruption of the FOG-1 gene markedly inhibits erythroid development, causing embryonic death from severe anemia. Detailed study of these animals and FOG-1 $1^{-1-}$ ES cells also demonstrated an early block to megakaryocytic development with no identifiable precursors. FOG-1 does not appear to bind DNA directly but, rather, associates with target genes indirectly through interactions with GATA proteins (Figure $3)$. FOG-1 is the only protein of its kind expressed in the erythromegakaryocytic lineages. The severity of the megakaryocyte defect in the FOG-1 $1^{-/-}$mouse suggests that most or all critical GATA-1and GATA-2-related activities require interactions with FOG-1. In support of this, GATA-1 and FOG-1 synergistically enhance the expression of the megakaryocyte-specific $\alpha_{\text {IIb }}$ gene $(80,81)$. These observations have been expanded to show that direct contact is needed between the N-terminal zinc finger of GATA-1 and FOG-1. This synergism applies to multiple megakaryocyte-specific genes and also involves a specific Ets family transcription factor (see below). The N-terminus of FOG-1 plays a unique, nonredundant role in megakaryocyte-specific expression (82), possibly through its ability to recruit the corepressor complex NuRD (83). The clinical importance of the GATA-1/FOG-1 interaction in megakaryopoiesis is demonstrated by the identification of patients with $\mathrm{X}$-linked thrombocytopenia and variable anemia who have GATA-1 mutations that impair FOG-1 binding (84-86) (Figure 2).

An important role for GATA-1 in regulating the maturation and proliferation of megakaryocyte progenitors is further evidenced by the recent discovery of acquired somatic mutations associated with both megakaryoblastic leukemia and transient myeloproliferative syndrome in infants with Down syndrome $(87,88)$. These mutations occur in the first coding exon, causing early termination and production of a truncated protein, termed GATA- $1^{\text {Short }}$, via translation initiation from a downstream internal methionine. This mutant form of GATA- 1 may act as a dominant oncogene by specifically stimulating the proliferation of fetal megakaryocyte progenitors (19).

Fli-1 and TEL. The proximal promoters of many megakaryocytespecific genes contain tandem-binding sites for GATA and Ets proteins, suggesting functional interactions between these 2 classes of 
transcription factors (71-73) (Figure 3). The Ets family is diverse with at least 30 members, all sharing an Ets-binding domain that recognizes a GGAA core sequence (89). Numerous Ets members are present in primary megakaryocytes and/or megakaryocytic cell lines (90-92). While a number of reports suggest a function for Ets-1 in megakaryopoiesis (93), the clearest story of a common transcriptional regulator appears to be that for Fli-1, an Ets transcription factor initially recognized to be important for $\mathrm{T}$ cell differentiation (94) and early hematopoiesis/vasculogenesis (95) (Figure 2). GATA-1/FOG-1 synergy for many megakaryocyte-specific genes appears to involve Fli-1 (81), and Fli-1 binds to the proximal promoter of these genes in vivo. The molecular basis of this synergy is still not fully known, but Fli-1 does bind GATA-1 (96) (Figure $3)$. In undifferentiated hematopoietic cell lines, overexpressed Fli-1 can induce megakaryocytic features (91). Moreover, Fli1 gene-disrupted mice either have abnormal megakaryocytes with thrombocytopenia (97) or fail to develop recognizable megakaryocytes (98), depending on the size of the Fli-1 gene deletion. Fli-1 expression also inhibits erythroid differentiation (99). Thus, Fli-1 may be a lineage-determining factor for megakaryocyte development. Hemizygous deficiency of Fli-1 expression causes thrombocytopenia associated with abnormal megakaryocytes in patients with Paris-Trousseau syndrome (100).

TEL, or ETV6, another Ets protein in the pointed domain subfamily, is closely related to Fli-1 and may also function in megakaryocytopoiesis (101). The pointed domain of TEL is a short $\mathrm{N}$-terminal domain involved in self-oligomerization. Like Fli-1, TEL is important in early HSCs (102) (Figure 2), and TEL overexpression can drive megakaryocyte differentiation of hematopoietic cell lines (92). Remarkably, conditional disruption of the TEL gene demonstrated a unique, nonredundant role for TEL in megakaryopoiesis (103). Specifically, loss of TEL in the erythromegakaryocyte lineage results in large, highly proliferative megakaryocytes and mild thrombocytopenia. This phenotype resembles that of GATA-1 deficiency and also has overlapping features with the NF-E2 knockout phenotype described below.

NF-E2. NF-E2 is a hematopoietic-specific transcription factor consisting of a tissue-specific p45 leucine zipper-containing subunit dimerized with a ubiquitous p18 subunit (104). In vitro studies suggested an important role for NF-E2 erythroid gene expression. Surprisingly, Nfe2-null mice do not develop anemia but, rather, exhibit severe thrombocytopenia with a marrow containing excessive immature, dysplastic megakaryocytes (15) (Figure 2). The molecular basis for this effect on megakaryocyte differentiation and platelet release has yet to be resolved. It may be that intracellular signaling pathways related to Rab27b (105) or cytoskeletal proteins (106) are underexpressed in the absence of p45, causing impaired proplatelet formation and platelet release.

$S C L$. SCL, or TAL1, is a basic helix-loop-helix transcription factor, initially identified in human $\mathrm{T}$ cell leukemias with multilineage characteristics (107), and has also been implicated in the earliest stages of hematopoiesis/vasculogenesis in the mouse embryo $(108,109)$ (Figure 2). LacZ knock-in studies suggest that SCL is expressed in myeloid, lymphoid, erythroid, and megakaryocytic lineages (110). In spite of this widespread expression, conditional disruption of the TAL1 gene in late-stage hematopoiesis demonstrated an absolute requirement only in the erythromegakaryocytic lineages of the yolk sac and fetal liver (111).

RUNX1. RUNX1 is a hematopoietic/vasculogenic-specific protein first noted because of its involvement in several leukemic chromosomal translocations, particularly $\mathrm{t}(8 ; 21)$, which generates the AML1-ETO fusion protein (112). RUNX1 is a heterodimer of core-binding factor $\alpha-2$ (CBFA2) that binds DNA and the $\beta$ subunit CBFB, which does not directly bind DNA (113). RUNX1 was initially thought to be solely involved in myeloid differentiation (114), but studies on targeting of the CBFA2 gene demonstrated an essential role for RUNX1 in early hematopoiesis and vasculogenesis $(115,116)$. A unique role for RUNX1 in adult megakaryopoiesis was established when a rare, dominantly inherited thrombocytopenia associated with an increased risk of developing acute myeloblastic leukemia was shown to be due to haploinsufficiency of CBFA2 $(117,118)$ (Figure 2). A role for CBFA2 in adult megakaryopoiesis was also confirmed in mice (119). RUNX1 appears to interact with GATA-1 (120) (Figure 3), and overexpression of RUNX1 can drive hematopoietic cell lines into a megakaryocytic phenotype (121), suggesting a role in lineage determination.

\section{Conclusions}

Mechanisms that regulate formation of the erythromegakaryocytic precursor and its commitment to unilineage megakaryocyte development are active areas of investigation. The discovery of new cytokines and transcription factors associated with megakaryopoiesis has enhanced our understanding of normal platelet development and human thrombocytopenias. One current challenge is to better define the developmental pathways though which MEPs and megakaryocytes arise from HSCs. For example, a recent finding that the onset of Flt 3 receptor expression in early hematopoiesis coincides with loss of erythromegakaryocytic capacity suggests novel pathways for MEP formation.

Additionally, comparative studies of developmental hematopoiesis in embryos and adults should extend our understanding of normal and pathologic megakaryopoiesis at distinct developmental stages. In addition, recent studies support a central role for multifactor transcriptional complexes containing GATA-1, FOG-1, and Fli-1 in terminal megakaryocytic differentiation. Formation and regulation of these complexes may illustrate a mechanism that initiates megakaryocyte commitment from the MEP. Further studies into all of these various areas of megakaryopoiesis promises to provide new insights into numerous hematopoietic disorders and may also have broader clinical applications by elucidating novel strategies to regulate platelet count and/or platelet thrombogenicity.

Address correspondence to: Mortimer Poncz, Children's Hospital of Philadelphia, 34th Street and Civic Center Boulevard, Abramson Research Center Room 317, Philadelphia, Pennsylvania 19104, USA. Phone: (215) 590-3574; Fax: (267) 426-5467; E-mail: poncz@email.chop.edu.
1. Ogawa, M. 1993. Differentiation and proliferation
of hematopoietic stem cells. Blood. 81:2844-2853.
2. Spangrude, G.J., Heimfeld, S., and Weissman, I.L.
1988. Purification and characterization of mouse
hematopoietic stem cells. Science. 241:58-62.
3. Ikuta, K., and Weissman, I.L. 1992. Evidence that
hematopoietic stem cells express mouse c-kit but do not depend on steel factor for their generation. Proc. Natl. Acad. Sci. U. S. A. 89:1502-1506.

4. Li, C.L., and Johnson, G.R. 1995. Murine hematopoietic stem and progenitor cells. I. Enrichment and biologic characterization. Blood. 85:1472-1479.

5. Weissman, I.L., Anderson, D.J., and Gage, F. 2001 Stem and progenitor cells: origins, phenotypes, lineage commitments and transdifferentiations. Annu. Rev. Cell Dev. Biol. 17:387-403.

6. Rosnet, O., Marchetto, S., deLapeyriere, O., and Birnbaum, D. 1991. Murine Flt3, a gene encoding a novel tyrosine kinase receptor of the PDGFR/ CSF1R family. Oncogene. 6:1641-1650.

7. Adolfsson, J., et al. 2001. Upregulation of Flt3 
expression within the bone marrow Lin(-)Sca1(+) c-kit(+) stem cell compartment is accompanied by loss of self-renewal capacity. Immunity. 15:659-669.

8. Kanz, L., Straub, G., Bross, K.G., and Fauser, A.A 1982. Identification of human megakaryocytes derived from pure megakaryocytic colonies (CFU-M), megakaryocytic-erythroid colonies (CFU-M/E), and mixed hemopoietic colonies (CFU-GEMM) by antibodies against platelet associated antigens. Blut. 45:267-274.

9. Nakahata, T., Gross, A.J., and Ogawa, M. 1982. A stochastic model of self-renewal and commitment to differentiation of the primitive hemopoietic stem cells in culture. J. Cell. Physiol. 113:455-458.

10. Akashi, K., Traver, D., Miyamoto, T., and Weissman, I.L. 2000. A clonogenic common myeloid progenitor that gives rise to all myeloid lineages. Nature. 404:193-197.

11. Debili, N. 1996. Characterization of a bipotent erythro-megakaryocytic progenitor in human bone marrow. Blood. 88:1284-1296.

12. Adolfsson, J., et al. 2005. Identification of Flt3+ lympho-myeloid stem cells lacking erythro-megakaryocytic potential: a revised road map for adult blood lineage commitment. Cell. 121:295-306.

13. Nakorn, T.N., Miyamoto, T., and Weissman, I.L. 2003. Characterization of mouse clonogenic megakaryocyte progenitors. Proc. Natl. Acad. Sci. U. S. A 100:205-210.

14. Branehog, I., Ridell, B., Swolin, B., and Weinfeld, A. 1975. Megakaryocyte quantifications in relation to thrombokinetics in primary thrombocythaemia and allied diseases. Scand. J. Haematol. 15:321-332.

15. Shivdasani, R.A., et al. 1995. Transcription factor NF-E2 is required for platelet formation independent of the actions of thrombopoietin/MGDF in megakaryocyte development. Cell. 81:695-704.

16. Palis, J., and Koniski, A. 2004. Analysis of hematopoietic progenitors in the mouse embryo. Methods Mol. Med. 105:289-302.

17. al-Jefri, A.H., Dror, Y., Bussel, J.B., and Freedman, M.H. 2000. Thrombocytopenia with absent radii: frequency of marrow megakaryocyte progenitors, proliferative characteristics, and megakaryocyte growth and development factor responsiveness. Pediatr. Hematol. Oncol. 17:299-306.

18. Crispino, J.D. 2005. GATA1 mutations in Down syndrome: implications for biology and diagnosis of children with transient myeloproliferative disorder and acute megakaryoblastic leukemia. Pediatr. Blood Cancer. 44:40-44

19. Li, Z., et al. 2005. Developmental stage-selective effect of somatically mutated leukemogenic transcription factor GATA1. Nat. Genet. 37:613-619.

20. Sola, M.C. 2004. Evaluation and treatment of severe and prolonged thrombocytopenia in neonates. Clin. Perinatol. 31:1-14.

21. Cajano, A., and Polosa, P. 1950. Contribution to the study of the morphology of megakaryocytes and blood platelets with Feulgen's test. Haematologica. 34:1113-11121.

22. Long, M.W. 1998. Megakaryocyte differentiation events. Semin. Hematol. 35:192-199.

23. Odell, T.T., Jr., Jackson, C.W., and Gosslee, D.G. 1965. Maturation of rat megakaryocytes studied by microspectrophotometric measurement of DNA. Proc. Soc. Exp. Biol. Med. 119:1194-1199.

24. Kozar, K. 2004. Mouse development and cell proliferation in the absence of D-cyclins. Cell. 118:477-491

25. Geng, Y. 2003. Cyclin E ablation in the mouse. Cell. 114:431-443

26. Geddis, A.E., and Kaushansky, K. 2004. Megakaryocytes express functional Aurora-B kinase in endomitosis. Blood. 104:1017-1024.

27. Zhang, Y., et al. 2004. Aberrant quantity and localization of Aurora-B/AIM-1 and survivin during megakaryocyte polyploidization and the conse- quences of Aurora-B/AIM-1-deregulated expression. Blood. 103:3717-3726.

28. Schmitt, A., Guichard, J., Masse, J.M., Debili, N., and Cramer, E.M. 2001. Of mice and men: comparison of the ultrastructure of megakaryocytes and platelets. Exp. Hematol. 29:1295-1302.

29. Lefebvre, P., Winter, J.N., Meng, Y., and Cohen, I. 2000. Ex vivo expansion of early and late megakaryocyte progenitors. J. Hematother. Stem Cell Res. 9:913-921.

30. Jagadeeswaran, P., Sheehan, J.P., Craig, F.E., and Troyer, D. 1999. Identification and characterization of zebrafish thrombocytes. Br. J. Haematol. 107:731-738.

31. Gregory, M., and Jagadeeswaran, P. 2002. Selective labeling of zebrafish thrombocytes: quantitation of thrombocyte function and detection during development. Blood Cells Mol. Dis. 28:418-427.

32. Gordon, M.S., and Hoffman, R. 1992. Growth factors affecting human thrombocytopoiesis: potential agents for the treatment of thrombocytopenia. Blood. 80:302-307.

33. Vainchenker, W., Debili, N., Mouthon, M.A., and Wendling, F. 1995. Megakaryocytopoiesis: cellular aspects and regulation. Crit. Rev. Oncol. Hematol. 20:165-192.

34. Orazi, A., et al. 1996. Effects of recombinant human interleukin-11 (Neumega rhIL-11 growth factor) on megakaryocytopoiesis in human bone marrow. Exp. Hematol. 24:1289-1297.

35. Kaushansky, K. 2005. The molecular mechanisms that control thrombopoiesis. J. Clin. Invest. 115:3339-3347. doi:10.1172/JCI26674.

36. Methia, N., Louache, F., Vainchenker, W., and Wendling, F. 1993. Oligodeoxynucleotides antisense to the proto-oncogene c-mpl specifically inhibit in vitro megakaryocytopoiesis. Blood. 82:1395-1401

37. Bartley, T.D., et al. 1994. Identification and cloning of a megakaryocyte growth and development factor that is a ligand for the cytokine receptor $\mathrm{Mpl}$. Cell. 77:1117-1124.

38. Lok, S., et al. 1994. Cloning and expression of murine thrombopoietin cDNA and stimulation of platelet production in vivo. Nature. 369:565-568.

39. Kaushansky, K., et al. 1994. Promotion of megakaryocyte progenitor expansion and differentiation by the c-Mpl ligand thrombopoietin. Nature. 369:568-571.

40. de Sauvage, F.J., et al. 1994. Stimulation of megakaryocytopoiesis and thrombopoiesis by the c-Mpl ligand. Nature. 369:533-538

41. Gurney, A.L., Carver-Moore, K., de Sauvage, F.J., and Moore, M.W. 1994. Thrombocytopenia in c-mpl-deficient mice. Science. 265:1445-1447.

42. Alexander, W.S., Roberts, A.W., Nicola, N.A., Li, R., and Metcalf, D. 1996. Deficiencies in progenitor cells of multiple hematopoietic lineages and defective megakaryocytopoiesis in mice lacking the thrombopoietic receptor c-Mpl. Blood. 87:2162-2170.

43. Murone, M., Carpenter, D.A., and de Sauvage, F.J. 1998. Hematopoietic deficiencies in c-mpl and TPO knockout mice. Stem Cells. 16:1-6.

44. Debili, N., et al. 1995. The Mpl-ligand or thrombopoietin or megakaryocyte growth and differentiative factor has both direct proliferative and differentiative activities on human megakaryocyte progenitors. Blood. 86:2516-2525.

45. Young, J.C., et al. 1996. Thrombopoietin stimulates megakaryocytopoiesis, myelopoiesis, and expansion of CD34+ progenitor cells from single CD34+Thy-1+Lin- primitive progenitor cells. Blood. 88:1619-1631.

46. Basser, R. 2002. The impact of thrombopoietin on clinical practice. Curr. Pharm. Des. 8:369-377.

47. Jelkmann, W. 2001. The role of the liver in the production of thrombopoietin compared with erythropoietin. Eur. J. Gastroenterol. Hepatol. 13:791-801.
48. Kaushansky, K. 1997. Thrombopoietin: understanding and manipulating platelet production. Annu. Rev. Med. 48:1-11.

49. Guerriero, A., et al. 1997. Thrombopoietin is synthesized by bone marrow stromal cells. Blood. 90:3444-3455.

50. Ihara, K., et al. 1999. Identification of mutations in the c-mpl gene in congenital amegakaryocytic thrombocytopenia. Proc. Natl. Acad. Sci. U. S. A. 96:3132-3136.

51. van den Oudenrijn, S., et al. 2000. Mutations in the thrombopoietin receptor, $\mathrm{Mpl}$, in children with congenital amegakaryocytic thrombocytopenia. Br. J. Haematol. 110:441-448.

52. Ballmaier, M., et al. 2001. c-mpl mutations are the cause of congenital amegakaryocytic thrombocytopenia. Blood. 97:139-146.

53. Ballmaier, M., Germeshausen, M., Krukemeier, S., and Welte, K. 2003. Thrombopoietin is essential for the maintenance of normal hematopoiesis in humans: development of aplastic anemia in patients with congenital amegakaryocytic thrombocytopenia. Ann. N. Y. Acad. Sci. 996:17-25.

54. Ghilardi, N., and Skoda, R.C. 1999. A single-base deletion in the thrombopoietin (TPO) gene causes familial essential thrombocythemia through a mechanism of more efficient translation of TPO mRNA. Blood. 94:1480-1482.

55. Ding, J., et al. 2004. Familial essential thrombocythemia associated with a dominant-positive activating mutation of the c-MPL gene, which encodes for the receptor for thrombopoietin. Blood. 103:4198-4200.

56. Kaushansky, K. 2005. On the molecular origins of the chronic myeloproliferative disorders: it all makes sense. Blood. 105:4187-4190.

57. Barbui, T. 2004. The leukemia controversy in myeloproliferative disorders: is it a natural progression of disease, a secondary sequela of therapy, or a combination of both? Semin. Hematol. 41:15-17.

58. Wang, J.F., Liu, Z.Y., and Groopman, J.E. 1998. The alpha-chemokine receptor CXCR4 is expressed on the megakaryocytic lineage from progenitor to platelets and modulates migration and adhesion. Blood. 92:756-764.

59. Majka, M., et al. 2000. Stromal-derived factor 1 and thrombopoietin regulate distinct aspects of human megakaryopoiesis. Blood. 96:4142-4151.

60. Kowalska, M.A., et al. 1999. Megakaryocyte precursors, megakaryocytes and platelets express the HIV co-receptor CXCR4 on their surface: determination of response to stromal-derived factor-1 by megakaryocytes and platelets. Br. J. Haematol. 104:220-229.

61. Avecilla, S.T., et al. 2004. Chemokine-mediated interaction of hematopoietic progenitors with the bone marrow vascular niche is required for thrombopoiesis. Nat. Med. 10:64-71.

62. Lee, B., Ratajczak, J., Doms, R.W., Gewirtz, A.M., and Ratajczak, M.Z. 1999. Coreceptor/chemokine receptor expression on human hematopoietic cells: biological implications for human immunodeficiency virus-type 1 infection. Blood. 93:1145-1156.

63. Seibert, C., and Sakmar, T.P. 2004. Small-molecule antagonists of CCR5 and CXCR4: a promising new class of anti-HIV-1 drugs. Curr. Pharm. Des. 10:2041-2062.

64. Gewirtz, A.M., Calabretta, B., Rucinski, B., Niewiarowski, S., and Xu, W.Y. 1989. Inhibition of human megakaryocytopoiesis in vitro by platelet factor 4 (PF4) and a synthetic $\mathrm{COOH}$-terminal PF4 peptide. J. Clin. Invest. 83:1477-1486.

65. Gewirtz, A.M., et al. 1995. Chemokine regulation of human megakaryocytopoiesis. Blood. 86:2559-2567.

66. Eslin, D.E., et al. 2004. Transgenic mice studies demonstrate a role for platelet factor 4 in thrombosis: dissociation between anticoagulant and antithrombotic effect of heparin. Blood. 104:3173-3180. 
67. Kowalska, M.A., et al. 2000. Stromal cell-derived factor-1 and macrophage-derived chemokine: 2 chemokines that activate platelets. Blood. 96:50-57.

68. Clemetson, K.J., et al. 2000. Functional expression of CCR1, CCR3, CCR4, and CXCR4 chemokine receptors on human platelets. Blood. 96:4046-4054.

69. Weiss, M.J., and Orkin, S.H. 1995. GATA transcription factors: key regulators of hematopoiesis. Exp. Hematol. 23:99-107.

70. Zon, L.I., et al. 1993. Expression of mRNA for the GATA-binding proteins in human eosinophils and basophils: potential role in gene transcription. Blood. 81:3234-3241.

71. Lemarchandel, V., Ghysdael, J., Mignotte, V., Rahuel, C., and Romeo, P.H. 1993. GATA and Ets cis-acting sequences mediate megakaryocyte-specific expression. Mol. Cell. Biol. 13:668-676.

72. Martin, F., Prandini, M.H., Thevenon, D., Marguerie, G., and Uzan, G. 1993. The transcription factor GATA-1 regulates the promoter activity of the platelet glycoprotein IIb gene. J. Biol. Chem. 268:21606-21612.

73. Deveaux, S., et al. 1996. Analysis of the thrombopoietin receptor (MPL) promoter implicates GATA and Ets proteins in the coregulation of megakaryocyte-specific genes. Blood. 87:4678-4685.

74. Ludlow, L.B., et al. 1996. Identification of a mutation in a GATA binding site of the platelet glycoprotein Ibbeta promoter resulting in the BernardSoulier syndrome. J. Biol. Chem. 271:22076-22080.

75. Fujiwara, Y., Browne, C.P., Cunniff, K., Goff, S.C., and Orkin, S.H. 1996. Arrested development of embryonic red cell precursors in mouse embryos lacking transcription factor GATA-1. Proc. Natl. Acad. Sci. U. S. A. 93:12355-12358.

76. Shivdasani, R.A., Fujiwara, Y., McDevitt, M.A., and Orkin, S.H. 1997. A lineage-selective knockout establishes the critical role of transcription factor GATA-1 in megakaryocyte growth and platelet development. EMBO J. 16:3965-3973.

77. Tsai, F.Y., et al. 1994. An early haematopoietic defect in mice lacking the transcription factor GATA-2. Nature. 371:221-226.

78. Fujiwara, Y., Chang, A.N., Williams, A.M., and Orkin, S.H. 2004. Functional overlap of GATA-1 and GATA-2 in primitive hematopoietic development. Blood. 103:583-585.

79. Tsang, A.P., et al. 1997. FOG, a multitype zinc finger protein, acts as a cofactor for transcription factor GATA-1 in erythroid and megakaryocytic differentiation. Cell. 90:109-119.

80. Gaines, P., Geiger, J.N., Knudsen, G., Seshasayee, D., and Wojchowski, D.M. 2000. GATA-1- and FOGdependent activation of megakaryocytic alpha IIB gene expression. J. Biol. Chem. 275:34114-34121.

81. Wang, X., et al. 2002. Control of megakaryocyte-specific gene expression by GATA-1 and FOG-1: role of Ets transcription factors. EMBO J. 21:5225-5234.

82. Cantor, A.B., Katz, S.G., and Orkin, S.H. 2002. Distinct domains of the GATA-1 cofactor FOG-1 differentially influence erythroid versus megakaryocytic maturation. Mol. Cell. Biol. 22:4268-4279.

83. Hong, W., et al. 2005. FOG-1 recruits the NuRD repressor complex to mediate transcriptional repression by GATA-1. EMBO J. 24:2367-2378.

84. Nichols, K.E., et al. 2000. Familial dyserythropoietic anaemia and thrombocytopenia due to an inherited mutation in GATA1. Nat. Genet. 24:266-270.

85. Yu, C., et al. 2002. X-linked thrombocytopenia with thalassemia from a mutation in the amino finger of GATA- 1 affecting DNA binding rather than FOG-1 interaction. Blood. 100:2040-2045.
86. Mehaffey, M.G., Newton, A.L., Gandhi, M.J., Crossley, M., and Drachman, J.G. 2001. X-linked thrombocytopenia caused by a novel mutation of GATA-1. Blood. 98:2681-2688.

87. Wechsler, J., et al. 2002. Acquired mutations in GATA1 in the megakaryoblastic leukemia of Down syndrome. Nat. Genet. 32:148-152.

88. Greene, M.E., et al. 2003. Mutations in GATA1 in both transient myeloproliferative disorder and acute megakaryoblastic leukemia of Down syndrome. Blood Cells Mol. Dis. 31:351-356.

89. Oikawa, T., and Yamada, T. 2003. Molecular biology of the Ets family of transcription factors. Gene. 303:11-34.

90. Terui, K., et al. 2000. Expression of transcription factors during megakaryocytic differentiation of CD34+ cells from human cord blood induced by thrombopoietin. Tohoku J. Exp. Med. 192:259-273.

91. Athanasiou, M., et al. 1996. Increased expression of the ETS-related transcription factor FLI-1/ERGB correlates with and can induce the megakaryocytic phenotype. Cell Growth Differ. 7:1525-1534.

92. Sakurai, T., et al. 2003. Effects of overexpression of the Ets family transcription factor TEL on cell growth and differentiation of K562 cells. Int. J. Oncol. 22:1327-1333.

93. Jackers, P., Szalai, G., Moussa, O., and Watson, D.K. 2004. Ets-dependent regulation of target gene expression during megakaryopoiesis. J. Biol. Chem. 279:52183-52190.

94. Anderson, M.K., Hernandez-Hoyos, G., Diamond, R.A., and Rothenberg, E.V. 1999. Precise developmental regulation of Ets family transcription factors during specification and commitment to the T cell lineage. Development. 126:3131-3148.

95. Brown, L.A., et al. 2000. Insights into early vasculogenesis revealed by expression of the ETS-domain transcription factor Fli-1 in wild-type and mutant zebrafish embryos. Mech. Dev. 90:237-252.

96. Eisbacher, M., et al. 2003. Protein-protein interaction between Fli-1 and GATA-1 mediates synergistic expression of megakaryocyte-specific genes through cooperative DNA binding. Mol. Cell. Biol. 23:3427-3441.

97. Hart, A., et al. 2000. Fli-1 is required for murine vascular and megakaryocytic development and is hemizygously deleted in patients with thrombocytopenia. Immunity. 13:167-177.

98. Kawada, H., et al. 2001. Defective megakaryopoiesis and abnormal erythroid development in Fli-1 genetargeted mice. Int. J. Hematol. 73:463-468.

99. Athanasiou, M., Mavrothalassitis, G., Sun-Hoffman, L., and Blair, D.G. 2000. FLI-1 is a suppressor of erythroid differentiation in human hematopoietic cells. Lenkemia. 14:439-445.

100. Raslova, H., et al. 2004. FLI1 monoallelic expression combined with its hemizygous loss underlies Paris-Trousseau/Jacobsen thrombopenia. J. Clin. Invest. 114:77-84. doi:10.1172/JCI200421179.

101. Mackereth, C.D., et al. 2004. Diversity in struc ture and function of the Ets family PNT domains. J. Mol. Biol. 342:1249-1264.

102.Wang, L.C., et al. 1998. The TEL/ETV6 gene is required specifically for hematopoiesis in the bone marrow. Genes Dev. 12:2392-2402.

103. Hock, H. et al. 2004. Tel/Etv6 is an essential and selective regulator of adult hematopoietic stem cell survival. Genes Dev. 18:2336-2341.

104.Andrews, N.C., Erdjument-Bromage, H., Davidson, M.B., Tempst, P., and Orkin, S.H. 1993. Erythroid transcription factor NF-E2 is a haematopoietic-specific basic-leucine zipper protein. Nature.
362:722-728.

105. Tiwari, S., et al. 2003. A role for Rab27b in NF-E2dependent pathways of platelet formation. Blood. 102:3970-3979.

106.Lecine, P., Italiano, J.E., Jr., Kim, S.W., Villeval, J.L., and Shivdasani, R.A. 2000. Hematopoietic-specific beta 1 tubulin participates in a pathway of platelet biogenesis dependent on the transcription factor NF-E2. Blood. 96:1366-1373.

107. Aplan, P.D., Lombardi, D.P., and Kirsch, I.R. 1991. Structural characterization of SIL, a gene frequently disrupted in T-cell acute lymphoblastic leukemia. Mol. Cell. Biol. 11:5462-5469.

108.Shivdasani, R.A., Mayer, E.L., and Orkin, S.H. 1995. Absence of blood formation in mice lacking the T-cell leukaemia oncoprotein tal-1/SCL. Nature. 373:432-434

109.Visvader, J.E., Fujiwara, Y., and Orkin, S.H. 1998 Unsuspected role for the T-cell leukemia protein SCL/tal-1 in vascular development. Genes Dev. 12:473-479.

110.Elefanty, A.G., et al. 1998. Characterization of hematopoietic progenitor cells that express the transcription factor SCL, using a lacZ "knock-in" strategy. Proc. Natl. Acad. Sci. U. S. A. 95:11897-11902.

111.Schlaeger, T.M., Mikkola, H.K., Gekas, C., Helgadottir, H.B., and Orkin, S.H. 2005. Tie2Cre-mediated gene ablation defines the stem-cell leukemia gene (SCL/tal1)-dependent window during hematopoietic stem-cell development. Blood. 105:3871-3874.

112.Nucifora, G., and Rowley, J.D. 1995. AML1 and the $8 ; 21$ and $3 ; 21$ translocations in acute and chronic myeloid leukemia. Blood. 86:1-14.

113.Speck, N.A., et al. 1999. Core-binding factor: a central player in hematopoiesis and leukemia. Cancer Res. 59(Suppl. 7):1789s-1793s.

114.Zhang, D.E., et al. 1996. Function of PU.1 (Spi-1), C/EBP, and AML1 in early myelopoiesis: regulation of multiple myeloid CSF receptor promoters. Curr. Top. Microbiol. Immunol. 211:137-147.

115.Takakura, N., et al. 2000. A role for hematopoietic stem cells in promoting angiogenesis. Cell. 102:199-209.

116. North, T., et al. 1999. Cbfa2 is required for the formation of intra-aortic hematopoietic clusters. Development. 126:2563-2575.

117.Song, W.J., et al. 1999. Haploinsufficiency of CBFA2 causes familial thrombocytopenia with propensity to develop acute myelogenous leukaemia. Nat. Genet. 23:166-175.

118. Michaud, J., et al. 2002. In vitro analyses of known and novel RUNX1/AML1 mutations in dominant familial platelet disorder with predisposition to acute myelogenous leukemia: implications for mechanisms of pathogenesis. Blood. 99:1364-1372.

119.Ichikawa, M., et al. 2004. AML-1 is required for megakaryocytic maturation and lymphocytic differentiation, but not for maintenance of hematopoietic stem cells in adult hematopoiesis. Nat. Med. 10:299-304.

120.Elagib, K.E., et al. 2003. RUNX1 and GATA-1 coexpression and cooperation in megakaryocytic differentiation. Blood. 101:4333-4341.

121.Niitsu, N., et al. 1997. AML1a but not AML1b inhibits erythroid differentiation induced by sodium butyrate and enhances the megakaryocytic differentiation of K562 leukemia cells. Cell Growth Differ. 8:319-326.

122. Westendorf, J.J., and Hiebert, S.W. 1999. Mammalian runt-domain proteins and their roles in hematopoiesis, osteogenesis, and leukemia [review]. J. Cell. Biochem. 75(Suppl.):51-58. 